G. C. Sloan, et al.

Science 323, 353 (2009);

DOI: $10.1126 /$ science.1165626

This copy is for your personal, non-commercial use only.

If you wish to distribute this article to others, you can order high-quality copies for your colleagues, clients, or customers by clicking here.

Permission to republish or repurpose articles or portions of articles can be obtained by following the guidelines here.

The following resources related to this article are available online at www.sciencemag.org (this information is current as of February 8, 2010 ):

Updated information and services, including high-resolution figures, can be found in the online version of this article at:

http://www.sciencemag.org/cgi/content/full/323/5912/353

This article cites $\mathbf{4 7}$ articles, 1 of which can be accessed for free:

http://www.sciencemag.org/cgi/content/full/323/5912/353\#otherarticles

This article has been cited by 1 article(s) on the ISI Web of Science.

This article appears in the following subject collections:

Astronomy

http://www.sciencemag.org/cgi/collection/astronomy 


\section{Dust Formation in a Galaxy with Primitive Abundances}

\author{
G. C. Sloan, ${ }^{1 *}$ M. Matsuura, ${ }^{2,3}$ A. A. Zijlstra, ${ }^{4}$ E. Lagadec, ${ }^{4}$ M. A. T. Groenewegen, ${ }^{5}$ \\ P. R. Wood, ${ }^{6}$ C. Szyszka, ${ }^{4}$ ]. Bernard-Salas, ${ }^{1}$ ]. Th. van Loon ${ }^{7}$
}

Interstellar dust plays a crucial role in the evolution of galaxies. It governs the chemistry and physics of the interstellar medium. In the local universe, dust forms primarily in the ejecta from stars, but its composition and origin in galaxies at very early times remain controversial. We report observational evidence of dust forming around a carbon star in a nearby galaxy with a low abundance of heavy elements, 25 times lower than the solar abundance. The production of dust by a carbon star in a galaxy with such primitive abundances raises the possibility that carbon stars contributed carbonaceous dust in the early universe.

$\mathrm{D}$ ust is an important astrophysical constituent. It regulates the cooling of the interstellar medium, attenuates light emitted by galaxies, and fragments collapsing molecular clouds into intermediate- and low-mass stars. The detection of dust in the early universe $(1,2)$ raises questions about its composition and origin. Primordial dust may differ substantially from dust in the Milky Way because it formed when the abundances of refractory elements were low. Polycyclic aromatic hydrocarbons (PAHs), a carbon-rich dust component, have been detected as far back as a redshift of 2.7 , or 2.5 billion years after the Big Bang (3), but galaxies with low metallicities (that is, with little enrichment from elements heavier than helium) have a deficit in PAH emission $(4,5)$. The $2200 \AA$ bump seen in ultraviolet extinction curves and associated with carbonaceous interstellar dust is also largely absent in both metalpoor galaxies (6) and distant quasars (7). The extinction curves of these galaxies still require carbon-rich dust (8), but the nature of that carbonrich dust is changing with metallicity.

The main sources of dust are the winds of evolved stars and supernovae (SNe). In the Milky Way, stars on the asymptotic giant branch (AGB) dominate the injection of dust into the interstellar medium (ISM) (9). The chemistry of this dust depends on the $\mathrm{C} / \mathrm{O}$ ratio in the photosphere of the star, which in turn depends on how much carbon produced by triple- $\alpha$ burning in the interior of the star has been dredged to the surface.

${ }^{1}$ Department of Astronomy, Cornell University, Ithaca, NY 14853-6801, USA. ${ }^{2}$ National Optical Astronomical Observatory of Japan, Osawa 2-21-1, Mitaka, Tokyo 181-8588, Japan. ${ }^{3}$ Department of Physics and Astronomy, University College London, Gower Street, London WC1E 6BT, UK. ${ }^{4}$ School of Physics and Astronomy, University of Manchester, Post Office Box 88, Manchester M60 1QD, UK. ${ }^{5}$ Royal Observatory of Belgium, Ringlaan 3, B-1180 Brussels, Belgium. ${ }^{6}$ Research School of Astronomy and Astrophysics, Australian National University, Cotter Road, Weston Creek Australian Capital Territory 2611, Australia. ${ }^{7}$ Astrophysics Group, Lennard-jones Laboratories, Keele University, Staffordshire ST5 5BG, UK.

*To whom correspondence should be addressed. E-mail: sloan@isc.astro.cornell.edu
The stable CO molecule will form until either $\mathrm{C}$ or $\mathrm{O}$ is exhausted. Solids form from the remainder, resulting in either oxygen-rich dust dominated by silicates or carbonaceous dust. Both observations $(10,11)$ and theory (12) show that the fraction of AGB stars that become carbonrich increases in more metal-poor systems.

There are reasons to expect reduced dust production at low metallicity. Radiation pressure on dust grains cannot drive the winds from oxygenrich AGB stars at metallicities below 0.1 of the solar value, which could lead to higher $\mathrm{SN}$ rates in primitive galaxies (13) and make $\mathrm{SNe}$ the dominant source of dust in the early universe (14). However, observational evidence that $\mathrm{SNe}$ produce sufficient dust, and produce more than they destroy, remains controversial $(15,16)$.

Studies with the Infrared Spectrograph (IRS) (17) on the Spitzer Space Telescope (18) of the

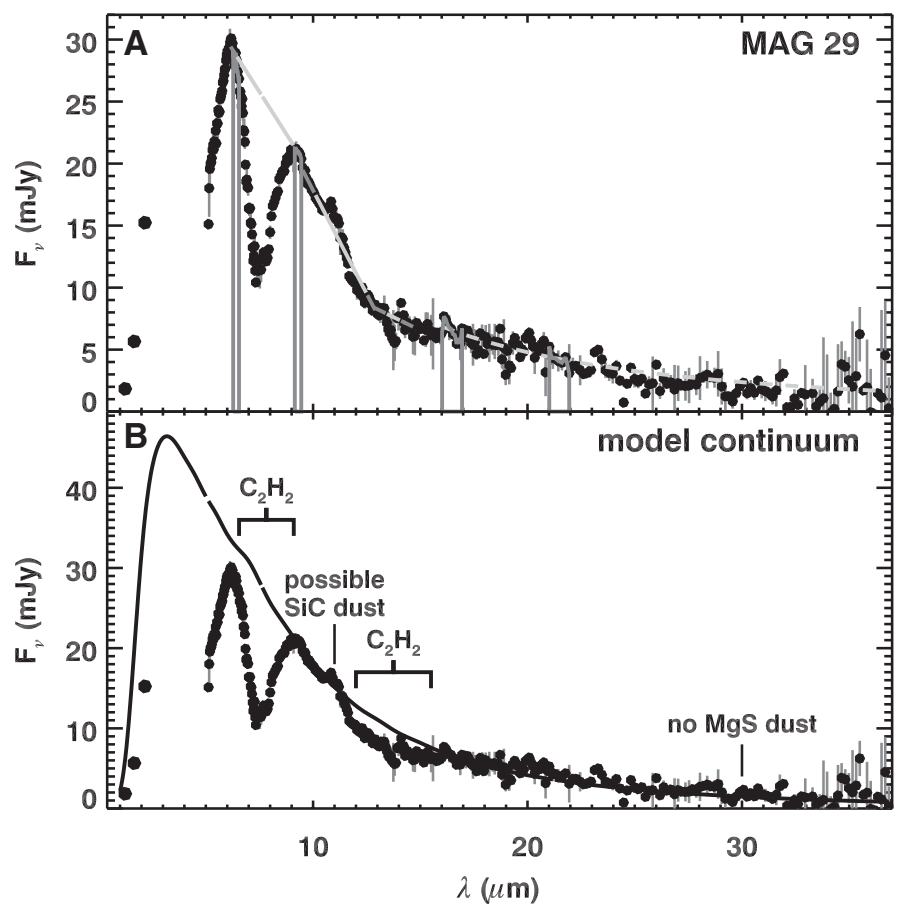

Large Magellanic Cloud (LMC) and Small Magellanic Cloud (SMC) (19-21) and the Fornax Dwarf Spheroidal (22) have revealed dust-forming carbon stars at metallicities as low as $\sim 0.1$ that of the Sun. As the metallicity of the sample decreases, the amount of dust produced by oxygenrich AGB stars decreases; but for carbon stars, the amount of dust remains unchanged $(23,24)$. Other properties of the outflows from carbon stars do depend on metallicity. As the metallicity of the sample drops, carbon stars produce less trace dust components such as $\mathrm{SiC}$ and $\mathrm{MgS}$, even though the production of amorphous carbon does not change. There is evidence for increasing abundances of acetylene $\left(\mathrm{C}_{2} \mathrm{H}_{2}\right)$ at lower metallicity. Acetylene is the fundamental building block of larger carbon-rich structures such as PAHs (25). Thus, mass loss and dust formation in carbon stars do not appear to depend on the abundances with which the star formed. Instead, free carbon (after the formation of $\mathrm{CO}$ ) needed by these stars to form dust is produced by the stars themselves and then dredged to the surface. It follows that even the most metal-poor carbon stars should produce dust.

To test this hypothesis, we observed a carbon star in the direction of the Sculptor Dwarf Spheroidal galaxy with the IRS on Spitzer. The Sculptor Dwarf is a satellite of the Milky Way, with a metallicity only 0.04 that of the Sun (26). A study of carbon stars in the Galactic Halo detected a candidate, MAG 29, in the field of the Sculptor Dwarf (27). This study assumed that the absolute magnitude in the narrow $K$ filter $\left(K_{\mathrm{s}}\right)$ of MAG 29 was -6.9 , but noted that it could be brighter by half a magnitude or more. The apparent $K_{\mathrm{s}}$ magnitude of MAG 29 is 11.60 (28). Thus, the assumed absolute $K_{\mathrm{s}}$ magnitude $\left(M_{\mathrm{K}}\right)$ implies a distance of $50 \mathrm{kpc}$, in the foreground of the

Fig. 1. The IRS spectrum of MAG 29. $F_{v}$, flux density per unit of frequency; $\lambda$, wavelength. The three points at 1.2 to $2.2 \mu \mathrm{m}$ represent older, ground-based 2MASS observations (28). (A) The Manchester method determines the [6.4] - [9.3] and $[16.5]-[21.5]$ colors (boxes), fits line segments over the $\mathrm{C}_{2} \mathrm{H}_{2}$ bands at 7.5 and $13.7 \mu \mathrm{m}$ and under the SiC emission feature at $\sim 11 \mu \mathrm{m}$ (solid lines), and extrapolates a Planck function from the [16.5] - [21.5] color under any possible MgS emission in the $30-\mu \mathrm{m}$ vicinity (dashed line). (B) A radiative transfer model of the dust continuum (solid curve). 
Sculptor Dwarf, which is $87 \mathrm{kpc}$ away (29). However, calibrations of near- and mid-infrared color magnitude relations (23) make MAG 29 a likely member of the Sculptor Dwarf. The $J-K_{\mathrm{s}}$ color (difference between apparent $J$ and $K_{\mathrm{s}}$ magnitudes) of MAG 29 is 3.24 (28). The linear relation between $J-K_{\mathrm{s}}$ and $M_{\mathrm{K}}$ implies $M_{\mathrm{K}}=$ $-7.90 \pm 0.51$ and a distance of $80 \pm 19 \mathrm{kpc}$. Measuring the magnitude of MAG 29 at 6.4 and $9.3 \mu \mathrm{m}([9.3])$ and applying the relation between [6.4] - [9.3] color and $M_{9.3}$ provides a second estimate of the distance. The [6.4] - [9.3] color is $0.494 \pm 0.012$, which gives an absolute magnitude at $9.3 \mu \mathrm{m}\left(M_{9.3}\right)=-11.38 \pm 0.59$. The apparent magnitude at $9.3 \mu \mathrm{m}$ is 8.35 , implying that the distance is $88 \pm 18 \mathrm{kpc}$. The two color-based distances are consistent with each other and the distance to the Sculptor Dwarf, but both are inconsistent with the previous provisional estimate of $50 \mathrm{kpc}$. Combining the two new distances gives $84 \pm 13 \mathrm{kpc}$, confirming the association between the star and the galaxy and making MAG 29 the most metal-poor, carbon-rich AGB star studied spectroscopically in the infrared.

The mid-infrared spectrum of MAG 29 shows a continuum, with strong molecular absorption bands (Fig. 1A). We analyzed the spectrum by applying the Manchester method (19), which uses the [6.4] - [9.3] color to diagnose the continuum temperature in this spectral region. Radiative transfer models show that this color varies linearly with the logarithm of the mass-loss rate (24). The calibration of [6.4] - [9.3] color to dust mass-loss rate (23), which assumes an outflow velocity of $10 \mathrm{~km} \mathrm{~s}^{-1}$, implies that MAG 29 is producing dust at the rate of $8 \times 10^{-9}$ solar masses $\left(M_{\odot}\right)$ year ${ }^{-1}$, with an uncertainty of $\sim 16 \%$.

We tested this conclusion by fitting a radiative transfer model (30) to the IRS spectrum and to the 2MASS data of Skrutskie et al. (28) (Fig. 1B). The 2MASS data were best fitted with a model in which the stellar temperature was $4000 \mathrm{~K}$, higher than found for Galactic mass-losing stars. Metalpoor AGB stars are bluer and hotter than their metal-rich counterparts (31). The dust shell around MAG 29 has a temperature of $1600 \mathrm{~K}$ at its inner radius. Although below the condensation temperature of amorphous carbon, it is higher than what is typically found for Galactic carbon stars, indicating that the dust in MAG 29 forms close to the star and that graphite may be a component. The fitted model implies that MAG 29 is producing dust at a rate of $2.5 \times 10^{-8} M_{\odot}$ year $^{-1}$. This value is larger than that estimated with the Manchester method by a factor of $\sim 3$ because the radiative transfer model leads to a higher outflow velocity ( 35 versus $10 \mathrm{~km} \mathrm{~s}^{-1}$ ). Assuming a gasto-dust ratio of 200 , the total mass-loss rate of

Fig. 2. The 7.5- $\mu \mathrm{m}$ absorption band in the spectrum of MAG 29, compared to the spectrum of MSX SMC 036, a typical carbon star in the SMC (19) (top) and to a synthetic absorption spectrum from acetylene $\left(\mathrm{C}_{2} \mathrm{H}_{2}\right)$ (bottom).

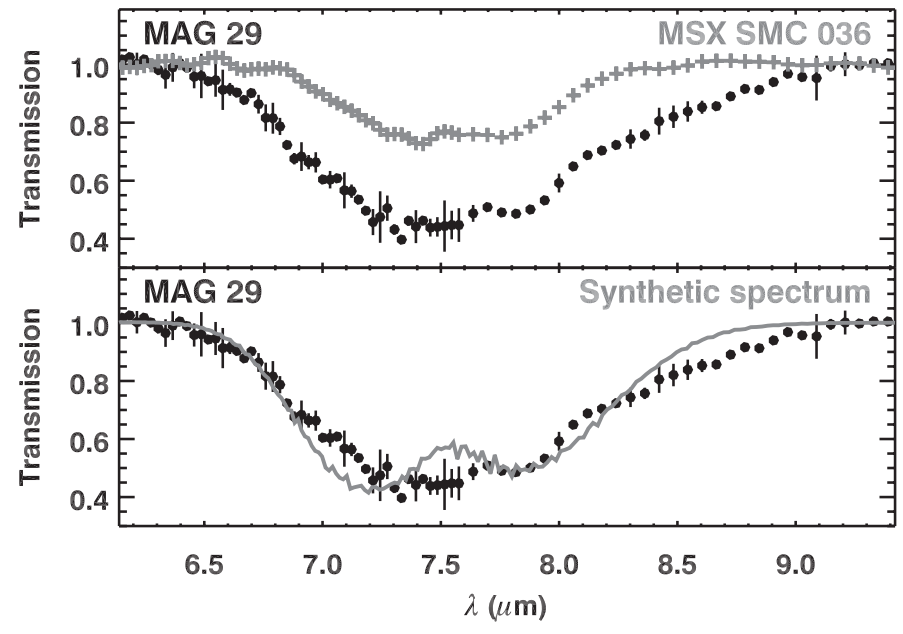

Fig. 3. The 11- to $16-\mu \mathrm{m}$ absorption band in the spectrum of MAG 29, plotted as transmission by dividing by the continuum as estimated from the radiative transfer model in Fig. 1 and compared to the IRS spectrum of IRAS 04496-6958 (33) (top) and a synthetic acetylene spectrum (bottom).

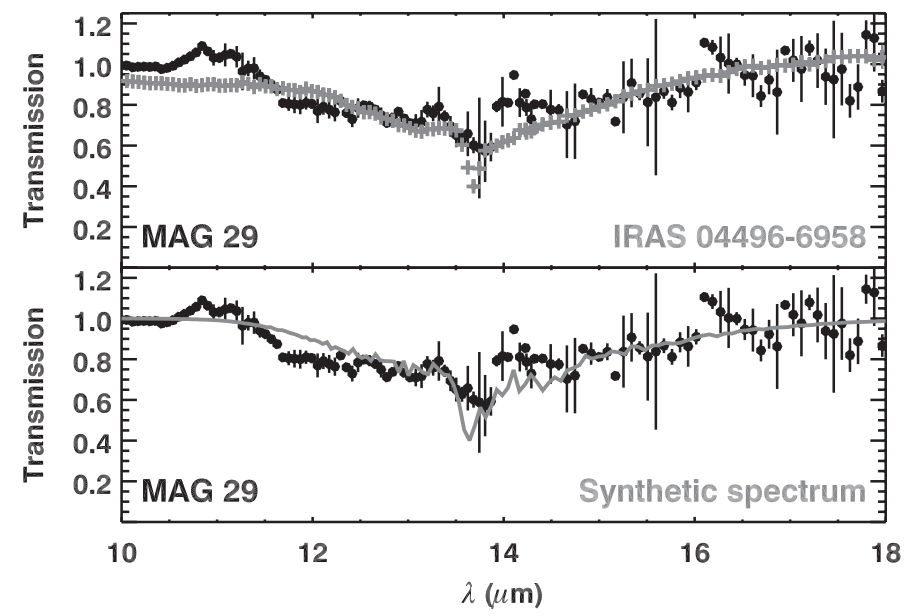

MAG 29 is in the range of $1.6 \times 10^{-6} M_{\odot}$ year $^{-1}$ to $4.9 \times 10^{-6} M_{\odot}$ year $^{-1}$. These results point to substantial mass loss and dust production.

In most carbon stars, amorphous carbon dominates the dust, but trace amounts of $\mathrm{SiC}$ and $\mathrm{MgS}$ produce emission features at $\sim 11.3$ and 26 to $30 \mu \mathrm{m}$, respectively. We used the Manchester method to fit a line segment to the continuum on either side of the $\mathrm{SiC}$ feature. We found a possible emission feature centered at $10.9 \mu \mathrm{m}$, similar to that found in two carbon stars in the SMC (19), with a strength of $3 \pm 1 \%$ that of the continuum. However, it is possible that what appears to be emission is simply continuum between a suspected absorption band at $10 \mu \mathrm{m}(20)$ and acetylene absorption between 11 and $16 \mu \mathrm{m}$. Therefore, the measured $\mathrm{SiC}$ strength is an upper limit of $4 \%$. The MgS emission extends beyond $37 \mu \mathrm{m}$, outside the wavelength coverage of the IRS. We extrapolated the continuum under the $\mathrm{MgS}$ emission feature by fitting a Planck function at 16.5 and $21.5 \mu \mathrm{m}$ and found no evidence of $\mathrm{MgS}$ emission.

The spectrum of MAG 29 shows a deep absorption band at $7.5 \mu \mathrm{m}$. It is substantially deeper and broader than the acetylene bands in carbon stars in the LMC (32). Thus, we modified the Manchester method by fitting a continuum at 6.14 to $6.44 \mu \mathrm{m}$ and 9.12 to $9.42 \mu \mathrm{m}$. The band's equivalent width is $0.79 \pm 0.02 \mu \mathrm{m}$, almost twice as strong as that measured in any other carbon star. We compared it to that of a typical carbon star in the SMC and to a synthetic spectrum of acetylene with an excitation temperature of $1750 \mathrm{~K}$ (Fig. 2). The synthetic spectrum reproduces the general shape of the band, demonstrating that acetylene is the primary absorber. The synthetic spectrum does not match the details of the absorption core, but it is limited by the available line lists, which do not include the higher-order transitions that will be populated at these high temperatures and column densities (33).

MAG 29 shows another acetylene feature centered at $13.7 \mu \mathrm{m}$. The Manchester method is not useful to study this feature because it measures the $\mathrm{Q}$ branch of this transition, but not the $\mathrm{P}$ and $\mathrm{R}$ branches that can extend from $\sim 11$ to $\sim 16 \mu \mathrm{m}$ (33). Matches between the data and a model of the continuum over this wavelength range show that the band's equivalent width is $0.990 \pm 0.022 \mu \mathrm{m}$. Only one carbon star, MSX SMC 093 in the SMC, has a stronger band (34), whereas IRAS 044966958 in the LMC, another carbon star, has a band of comparable strength (Fig. 3)

We compared the acetylene strength and the $\mathrm{SiC}$ strength of MAG 29 with those observed in Local Group samples (Fig. 4). We limited the comparison sample to those sources with colors similar to those of MAG 29; that is, those with $0.35 \leq[6.4]-[9.3] \leq 0.65$. The data for the $7.5-\mu \mathrm{m}$ feature and $\mathrm{SiC}$ strength are from previously published studies of the Magellanic Clouds $(19-21,23)$ and new analysis of spectra from the Fornax Dwarf (22). The 11- to $16-\mu \mathrm{m}$ data are taken directly from a recent study (34). We adopted $[\mathrm{Z} / \mathrm{H}]=0.00 \pm 0.10$ for the Galaxy $(\mathrm{Z}$ is the metal 
Fig. 4. The metallicity dependence of the acetylene equivalent widths at $7.5 \mu \mathrm{m}$ (top) and 11 to $16 \mu \mathrm{m}$ (middle) and the $\mathrm{SiC} /$ continuum flux ratio (bottom) for sources with $0.35 \leq[6.4]-[9.3] \leq$ 0.65 . The error bars are the standard deviation of the populations, except for MAG 29. We have considered the $\mathrm{SiC}$ strength in MAG 29 to be $0 \pm 4 \%$ of the continuum, although it could be as large as $3 \pm 1 \%$.

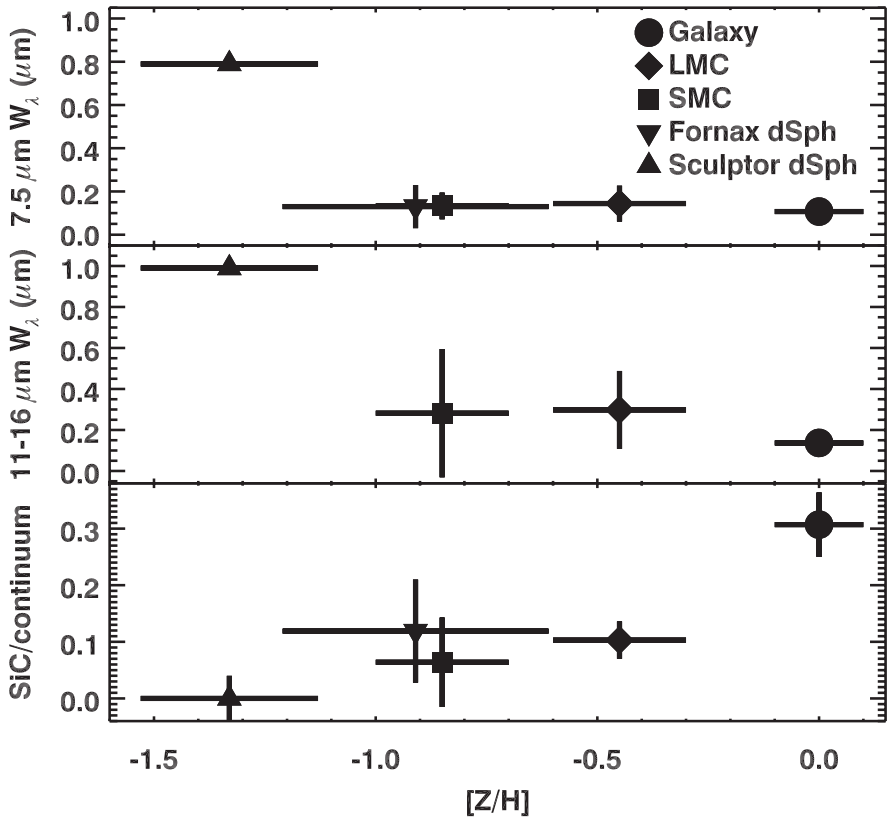

abundance), $-0.45 \pm 0.15$ for the $\mathrm{LMC}$, and $-0.85 \pm 0.15$ for the SMC (35). We adopted $[\mathrm{Z} / \mathrm{H}]=-1.33 \pm 0.20$ for the Sculptor Dwarf $(26)$ and $[\mathrm{Z} / \mathrm{H}]=0.93 \pm 0.30$ for Fornax [based on $[\mathrm{Fe} / \mathrm{H}]=-1.0 \pm 0.3(36)$ and assuming the same $[\alpha / \mathrm{Fe}]$ as for the Sculptor Dwarf]. The weak trend of increasing acetylene absorption with decreasing metallicity is more readily apparent when the data are examined over a larger color range (23). The spectrum of MAG 29 shows that the previously observed trends extend to metallicities as low as that of the Sculptor Dwarf.

In the Galactic environment, radiation pressure on carbon-rich dust can drive high mass loss from carbon stars (37). A carbon star self-produces its carbon via the triple- $\alpha$ reaction; if it can dredge up enough excess carbon, compared to oxygen, to allow the formation of carbonaceous dust, this high-mass-loss phase becomes inevitable (38). The spectrum of MAG 29 shows that metallicity is not a hurdle for the formation of dust around carbon stars. Metal-poor stars have an increased carbon dredge-up efficiency and lack the intrinsic oxygen that offsets dredged-up carbon, leading to a higher amount of excess carbon after the formation of $\mathrm{CO}(39,40)$.

Once enough time has elapsed after a galaxy has formed for stars to reach the AGB and dredge up carbon, its ISM should be seeded with carbonrich dust. Dust has been detected in galaxies to a redshift of 6.4 (1), or only 870 million years after the Big Bang. Recent modeling (41) shows that as soon as trace amounts of metals $\left(10^{-5}\right.$ of the solar amount) appear in the ISM, intermediatemass stars can form. Thus, precursors of carbon stars will form almost immediately after the formation of galaxies, which should have occurred by a redshift of $\sim 10$, or $\sim 480$ million years after the Big Bang (42). Thus, carbon stars have $\sim 390$ million years to evolve from their birth to the AGB. Models of stars at 0.01 solar metallicity (43) be- come carbon-rich over the full mass range studied $\left(2.5\right.$ to $\left.5 M_{\odot}\right)$. Similarly, all models from 2 to $6 M_{\odot}$ at half that metallicity also become carbonrich (44). These models will evolve from the zeroage main sequence to the AGB in only 70 million years for the most massive stars. The $3-M_{\odot}$ models require only 280 to 310 million years. These time scales are less than the 500- million-year time scale used previously to rule out carbon stars as contributors to the dust at these redshifts $(14,45)$.

How much dust carbon stars can contribute at these redshifts depends on variables that are more difficult to quantify. The amount of carbon formed and dredged up differs by an order of magnitude in the models referred to above. The star formation rate and the initial mass function will determine how many stars form in the appropriate mass range. The star formation rates needed for carbon stars to explain the dust observed at high redshift may be consistent with observed luminosities (46). All of these quantities need to be better understood before we can quantify how much dust carbon stars contributed in the early universe.

The relative contributions of $\mathrm{SNe}$ and carbon stars to dust in the early universe is a problem of great interest. Core-collapse $\mathrm{SNe}$ appear well before the first carbon stars, whereas type Ia SNe require white dwarfs, which appear only after AGB stars have evolved. Core-collapse $\mathrm{SNe}$ can produce both silicates and carbonaceous dust; it is difficult to determine which would dominate (47). Current measurements of the dust around observed $\mathrm{SNe}$ fall short of what is needed to account for the dust observed at high redshifts (45), but we lack direct observations of $\mathrm{SNe}$ at low metallicity. A recent study of dust extinction at a redshift of 6.2 found evidence for carbonaceous dust (48). In the light of the observations reported here, the presence of such dust could be explained, at least in part, by the mass loss from carbon stars in the early universe.

\section{References and Notes}

1. F. Bertoldi et al., Astron. Astrophys. 406, L55 (2003).

2. D. C. Hines et al., Astrophys. J. 641, L85 (2006).

3. H. I. Teplitz et al., Astrophys. J. 659, 941 (2007).

4. C. W. Engelbracht et al., Astrophys. J. 628, L29 (2005).

5. Y. Wu et al., Astrophys. J. 639, 157 (2006).

6. K. D. Gordon, G. C. Clayton, K. A. Misselt, A. U. Landolt, M. J. Wolff, Astrophys. J. 594, 279 (2003).

7. K. M. Pitman, G. C. Clayton, K. D. Gordon, Publ. Astron. Soc. Pac. 112, 537 (2000)

8. J. S. Mathis, Astrophys. J. 497, 824 (1998).

9. R. Gehrz, in Proceedings of IAU Symposium 135, L. ]. Allamandola, A. G. G. M. Tielens, Eds. (Kluwer Academic, Dordrecht, Netherlands, 1989), pp. 445-453.

10. B. M. Blanco, V. M. Blanco, M. F. McCarthy, Nature 271 , 638 (1978)

11. V. M. Blanco, B. M. Blanco, M. F. McCarthy, Astrophys. J. 242, 938 (1980)

12. A. Renzini, M. Voli, Astron. Astrophys. 94, 175 (1981).

13. G. H. Bowen, L. A. Willson, Astrophys. J. 375, L53 (1991).

14. E. Dwek, Astrophys. J. 501, 643 (1998).

15. B. E. K. Sugerman et al., Science 313, 196 (2006).

16. W. P. S. Meikle et al., Astrophys. J. 508, 106 (2007).

17. J. R. Houck et al., Astrophys. J. Suppl. 154, 18 (2004).

18. M. W. Werner et al., Astrophys. J. Suppl. 154, 1 (2004)

19. G. C. Sloan et al., Astrophys. J. 645, 1118 (2006).

20. A. A. Zijlstra et al., Mon. Not. R. Astron. Soc. 370, 1961 (2006).

21. E. Lagadec et al., Mon. Not. R. Astron. Soc. 376, 1270 (2007).

22. M. Matsuura et al., Mon. Not. R. Astron. Soc. 382, 1889 (2007).

23. G. C. Sloan et al., Astrophys. ]. 686, 1056 (2008).

24. M. A. T. Groenewegen et al., Mon. Not. R. Astron. Soc. 376, 313 (2007)

25. M. Frenklach, E. D. Feigelson, Astrophys. J. 341, 372 (1989).

26. C. Babusiaux, G. Gilmore, M. Irwin, Mon. Not. R. Astron. Soc. 359, 985 (2005)

27. N. Mauron, M. Azzopardi, K. Gigoyan, T. R. Kendall, Astron. Astrophys. 418, 77 (2004).

28. M. F. Skrutskie et al., Astron. J. 131, 1163 (2006).

29. J. Kaluzny et al., Astron. Astrophys. 112, 407 (1995).

30. M. Elitzur, Ž. Ivezić, Mon. Not. R. Astron. Soc. 327, 403 (2001).

31. E. M. Levesque et al., Astrophys. J. 628, 973 (2005).

32. M. Matsuura et al., Mon. Not. R. Astron. Soc. 371, 415 (2006).

33. A. K. Speck et al., Astrophys. J. 650, 892 (2006).

34. J. M. Leisenring, F. Kemper, G. C. Sloan, Astrophys. J. 681, 1557 (2008).

35. J. Th. van Loon, Astron. Astrophys. 354, 125 (2000)

36. E. Tolstoy et al., Mon. Not. R. Astron. Soc. 327, 918 (2001).

37. P. Woitke, Astron. Astrophys. 460, L9 (2006).

38. E. Lagadec, A. A. Zijlstra, Mon. Not. R. Astron. Soc. 390, L59 (2008).

39. J. Th. van Loon, A. A. Zijlstra, M. A. T. Groenewegen, Astron. Astrophys. 346, 805 (1999).

40. M. Matsuura et al., Astron. Astrophys. 434, 691 (2005).

41. P. C. Clark, S. C. O. Glover, R. S. Klessen, Astrophys. J. 672, 757 (2008)

42. T. H. Greif, ]. L. Johnson, R. S. Klessen, V. Bromm, Mon. Not. R. Astron. Soc. 387, 1021 (2008).

43. P. Ventura, F. D'Antona, I. Mazzitelli, Astron. Astrophys. 393, 215 (2002)

44. F. Herwig, Astrophys. J. Suppl. 155, 651 (2004).

45. E. Dwek, F. Galliano, A. P. Jones, Astrophys. J. 662, 927 (2007).

46. H. L. Morgan, M. G. Edmunds, Mon. Not. R. Astron. Soc. 343, 427 (2003)

47. R. Schneider, A. Ferrara, R. Salvaterra, Mon. Not. R. Astron. Soc. 351, 1379 (2004)

48. R. Maiolino et al., Nature 431, 533 (2004).

49. Support for G.C.S. was provided by NASA through contract number 1257184 issued by the Jet Propulsion Laboratory, California Institute of Technology, under NASA contract 1407. The Australian Research Council provided support to P.R.W., and M.M. was supported by a Japan Society for the Promotion of Science fellowship.

8 September 2008; accepted 2 December 2008 10.1126/science. 1165626 